\title{
Botulinum A toxin treatment for detrusor-sphincter dyssynergia in spinal cord disease
}

\author{
H Petit $^{1}$, L Wiart ${ }^{2}$, E Gaujard ${ }^{3}$, F Le Breton ${ }^{1}$, JM Ferrière ${ }^{4}$, A Lagueny ${ }^{5}$, PA Joseph ${ }^{1}$ and M Barat ${ }^{1}$ \\ ${ }^{1}$ Service de rééducation fonctionnelle, Hôpital Pellegrin, Bordeaux, France, ${ }^{2}$ Centre de rééducation fonctionnelle La \\ Tour de Gassies, Bruges, ${ }^{3}$ Centre de rééducation fontionnelle Les Grand Chênes, Bordeaux, ${ }^{4}$ Service d'urologie, \\ Hôpital Pellegrin, Bordeaux, ${ }^{5}$ Service de neurologie, Hôpital Haut-Lévêque, Pessac
}

\begin{abstract}
We studied the efficacy of endoscopic injection of Botulinum A toxin (150 I.U. Dysport $\left.{ }^{\circledR}\right)$ in the treatment of detrusor-sphincter dyssynergia in 17 patients with spinal cord disease. One month after the injection, the postvoiding residual urine volume $(-176 \mathrm{ml}, P<0.001)$, the bladder pressure on voiding $(-19 \mathrm{~cm}$ water, $P<0.01)$, and the urethral pressure $(-24 \mathrm{~cm}$ water, $P<0.001)$ were significantly decreased. The modality of voiding was improved in 10 patients (ie micturition by suprapubic tapping was easier to induce, discontinuation of indwelling catheter use, or decrease in frequency of intermittent catheterizations). The tolerance of the treatment was excellent. The therapeutic effect lasted 2 to 3 months on the average. The low doses used in this study probably explain in part why the treatment sometimes failed. Botulinum A toxin could become an alternative treatment for detrusorsphincter dyssynergia in certain patients, notably in those who are refractory to sphincterotomy or in patients, such as those who are tetraplegic, and who are incapable of performing intermittent self-catheterization.
\end{abstract}

Keywords: spinal cord disease; detrusor-sphincter dyssynergia; botulinum toxin

\section{Introduction}

Detrusor-sphincter dyssynergia (DSD) represents a major cause of voiding dysfunction in those who have an upper motor neuron bladder abnormality. This dyssynergia can lead to chronic urinary retention, high intravesical pressure, chronic infections, vesicoureteral reflux and renal damage.

Oral treatments (adrenergic alpha antagonists, antispastic agents) for DSD have little effect. ${ }^{1}$ Intermittent self-catheterisation (ISC) is the principal treatment in selected patients, but remains impossible in tetraplegic patients, or in those with altered cerebellar function - which is frequently present in patients with multiple sclerosis. ISC may also be poorly tolerated locally (hematuria, urethral stricture) or psychologically. ${ }^{2}$ Sphincterotomy is effective but permanent incontinence may be induced and a high reoperation rate and long term failure has been described. $^{3,4}$ Sacral anterior root stimulators and posterior rhizotomy are mainly indicated for patients with complete neural deficits. ${ }^{5}$ Thus, there remain patients in which known treatments for DSD are insufficient or excessively invasive.

Botulinum A toxin (BTx) is an inhibitor of acetylcholine release at the neuromuscular junction and is used principally in the management of focal dystonia, spasms and spasticity., ${ }^{6,7}$ It was first

Correspondence: Dr H Petit, M.D. evaluated as a treatment for DSD by Dykstra et $a l^{8,9}$ in 16 patients, then by Schurch et al ${ }^{10,11}$ in 24 patients. These studies showed that BTx relaxes the external urethral sphincter (EUS) and reduces DSD in approximately $80 \%$ of cases. However, these authors used iterative injections (between one and five with an interval of 1 week between injections for Dykstra, and 1 month for Schurch) with various doses of BTx (between 100 and 720 I.U. of Botox $^{\circledR}$ BTx and 750 I.U. of Dysport ${ }^{\circledR}$ BTx) making comparisons and assessment of the cost/efficacy ratio of this expensive treatment difficult. We wished to determine if a lower dose of BTx would provide a therapeutic effect in certain patients, so that the cost might be reduced as much as possible. Thus, the object of our study was to assess the efficacy of a single cytoscopic injection of 150 I.U. BTx $\left(\right.$ Dysport $\left.^{\circledR}\right)$ for DSD in patients with spinal cord disease.

\section{Methods}

The inclusion criteria were a stable neurological status, the presence of an upper motor neuron type of bladder dysfunction, the existence of DSD defined as inappropriate contractions of the external urethral sphincter (EUS) coincident with detrusor contractions $^{\text {"12 }}$ and diagnosis by a urodynamic examination and by voiding cystourethrography, a postvoiding urine volume (PRUV) greater than $100 \mathrm{ml}$, and failure 
of oral treatment by adrenergic alpha-antagonists (alfuzosin) and antispastic agents (baclofen, dantrolene). Enlightened oral consent was obtained from each subject.

Exclusion criteria were pregnancy, allergy to the toxin, hemocoagulation abnormalities, myasthenia, treatment by aminoglycosides (the aminoglycosides have an effect on the myoneural junction and may be actually facilitating to BTx), inflammation or infection of the injection site, or alterations of the proximal urinary tract.

Endoscopic injection into the external urethral sphincter at three or four points was performred in all patients by the same coauthor of this report (JMF). This injection was performed under general anaesthesia in patients with incomplete cord lesions (to avoid painful phenomena when pain sensation was conserved) and under simple sedation (diazepam) in those with complete cord lesions. For the $24 \mathrm{~h}$ following the injection, the patients had an indwelling Foley catheter. All patients had an initial injection of 150 I.U. BTx (Dysport ${ }^{\circledR}$ ), diluted to $4 \mathrm{ml}$ with $0.9 \%$ saline solution (ie $1 \mathrm{ml}$ for each of the four points of injection into the striated urethral sphincter). We chose a procedure similar to that of Dykstra ${ }^{9}$ diluting BTx more than is generally recommended because we aimed to avoid excessive loss of the toxin linked to the technical constraints of the injection site (losses in the relatively long tubing of the cystoscope, and the possibility of toxin leakage into the urethral lumen by bleeding).

The pretherapeutic work-up included a complete medical history, a physical examination, a micturition diary, three PRUV measurements, a sample of the urine for culture, a voiding cystourethrography and a urodynamic examination.

Assessment was performed as follows. On day 15, PRUV was measured. On day 30, PRUV and a micturition diary were recorded, the patient's personal opinion was quantified on two analogue visual scales: Do you find this treatment to be efficacious? from 0 (no efficacy) to 10 (maximal efficacy), Have you tolerated this treatment well? from 0 (very poor tolerance) to 10 (very good tolerance), side effects, when present, were noted, and a urodynamic examination was performed. On day 60 and the subsequent assessment dates, PRUV was measured and a urodynamic examination carried out once a month until the reappearance of the former conditions. If the first injection failed, a second injection of 250 I.U. was administered under the same conditions 2 months later. If the therapeutic effect persisted longer than 4 months, PRUV and a urodynamic examination were recorded at 6 months and 1 year.

Urodynamic examinations were performed on patients reclined in a Dantec Urodyn 5500 apparatus using water for bladder filling. Bladder and urethral pressures were recorded simultaneously during continuous bladder filling at $50 \mathrm{ml} / \mathrm{min}$ with isotonic saline, and during uninhibited contractions or voiding (voluntary or by suprapubic tapping). Once in position, the urodynamic catheter was retained in place by external adhesive tape and the location of the microtransducers was verified by measuring pressures during coughing. Three urethral pressure profiles were also recorded. A transperineal electromyogram of the EUS was coupled with a urodynamic examination in seven of the patients before and 1 month after injection, the other 10 did not have an electromyogram. The principle parameters analyzed were the bladder pressure during inhibited bladder contractions or during voiding, urethral pressure profiles, and PRUV. Urine cultures were performed before each assessment. If infection was present, the urodynamic examination was performed after antimicrobial therapy.

Other therapeutic means that may have interfered with bladder functioning were discontinued during the trial except in four patients in whom antispastic or antidepressive medication was maintained at a constant dose throughout the study $(60 \mathrm{mg}$ : day baclofen in three cases, $6 \mathrm{mg} /$ day clonazepam in one case, $50 \mathrm{mg}$ /day clomipramine in one case). No search for anti-BTx antibodies was carried out.

Statistical analysis was performed using the nonparametric Wilcoxon test to compare values before and after injection of BTx.

\section{Results}

Seventeen patients were included between January and September 1995. All were male. Their average age was $35 \pm 13$ years (range: 22 to 63 ). Eight were tetraplegic and three paraplegic, resulting from trauma. Six patients had nontraumatic myelopathies: four idiopathic, one bilharzial and one by impingement. Seven patients had complete motor lesions. The average period of time between the occurrence of spinal cord lesions and inclusion in this trial was $36.4 \pm 56.5$ months.

Before treatment, five patients used an indwelling Foley catheter or had suprapubic drainage because of complete urinary retention or difficulties with intermittent catheterization; six others practised intermittent self-catheterization but wished to stop, two had intermittent catheterization, three voided using suprapubic tapping, and one voided using suprapubic tapping and Crede's manoeuvre. In our study, the three types of goal for treatment with BTx were thus discontinuation of indwelling drainage, discontinuation or diminution of intermittent catheterization, or improvement of tapping efficacy. Initial PRUV and urodynamic parameters are shown in Table 1.

After BTx injection, PRUV $(-176 \mathrm{ml})$ as well as urethral and bladder pressure on voiding $(-24$ and $-19 \mathrm{~cm}$ of water, respectively) decreased significantly on day 30 (Table 1). The mean score of efficacy attributed by the patients on an analogue scale was $5.3 \pm 3.8$ out of 10 . It was judged to be higher than seven by seven patients. The tolerance score was 
Table 1 PRUV, BPV and UP before and after treatment

\begin{tabular}{lccc}
\hline & $P R U V(\mathrm{ml})$ & BPV $($ cm.water $)$ & UP (cm.water) \\
& mean $(S D)$ & mean $(S D)$ & $(S D)$ \\
\hline Before treatment & $302(126)$ & $95(43)$ & $113(260$ \\
One month after treatment & $138(123)$ & $76(34)$ & $89(25)$ \\
$P$ value & $<0.001$ & $<0.01$ & $<0.001$ \\
\hline
\end{tabular}

PRUV: postvoiding residual urine volume, BPV: bladder pressure on voiding, UP: urethral pressure, SD: standard deviation

$9.1 \pm 1.1$ out of 10 . The side effects reported were one case of first-day urethral bleeding, the appearance of stress incontinence in two patients, and exacerbation of preexisting incontinence in three patients who were already using a condom catheter. The EMG aspect was modified after injection in only three patients in which there was noted to be a diminution of activity.

Use of a Foley catheter or suprapubic drainage was replaced in two patients by suprapubic tapping and in one by suprapubic tapping and Crede's manoeuvre. Four patients progressed from catheterization or selfcatheterization to suprapubic tapping. Among the four patients initially using suprapublic tapping, three had PRUVs less than $100 \mathrm{ml}$ and required less time tapping to induce voiding after treatment.

In summary, 10 patients were substantially improved by the injection of BTx. In two other patients, only the urodynamic parameters were noticeably modified, and in five others, there was no improvement after treatment.

Follow-up showed that the effect of BTx lasted from 2 to 3 months in three patients and 3 to 5 months in two others. In three patients lost to follow-up at 3 months, BTx was still effective after 60 days. In two patients, the efficacy of the treatment lasted for 1 year for the parameters 'PRUV' and 'bladder pressure on voiding', whereas 'urethral pressure' returned to initial values after 3 months in one and 6 months in the other. Among the seven patients who showed little or no improvement after the first injection, four accepted a second trial injection using a higher dose of BTx (250 I.U.). The second injection was effective in two patients.

A comparison of the patients who improved after injection to those who did not revealed that relatively poor bladder contractility (maximum bladder pressure less than $75 \mathrm{~cm}$ of water) tended to be more frequent in the unimproved patients (three out of seven compared to two out of 10 responders). A high initial PRUV (over $300 \mathrm{ml}$ ) did not appear to predispose to failure. Thus, among the 10 patients whose initial PRUV was between 300 and $500 \mathrm{ml}$, the treatment was effective in seven cases (Figures 1 and 2).

\section{Discussion}

This study shows that BTx reduces DSD and its attending urinary retention. DSD was improved in approximately $70 \%$ of our cases: 10 out of 17 patients

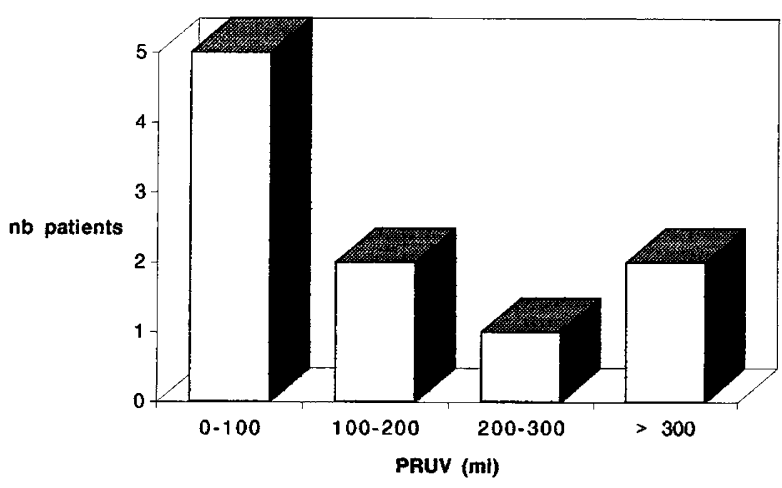

Figure 1 PRUV 1 month after treatment in the group of patients whose initial PRUV was higher than $300 \mathrm{ml}$. PRUV: postvoiding residual urine volume after treatment

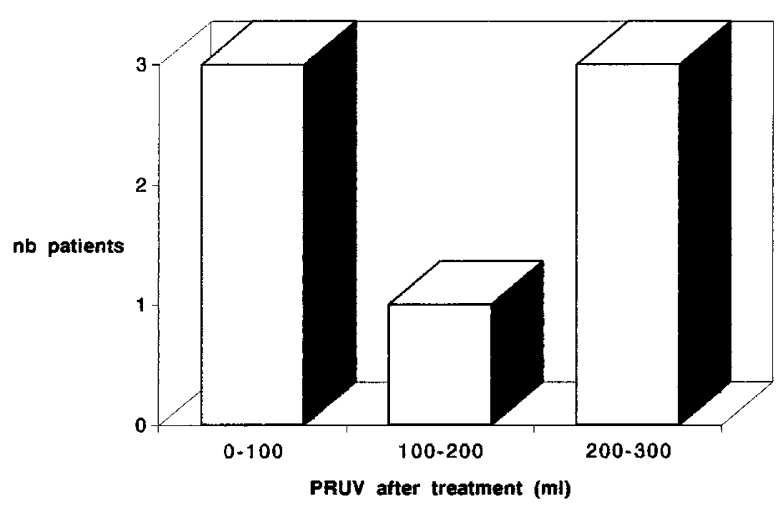

Figure 2 PRUV 1 month after treatment in the group of patients whose initial PRUV was between 100 and $300 \mathrm{ml}$. PRUV: postvoiding residual urine volume after treatment

had marked improvement in our study, and in two others the urodynamic anomalies regressed. The improvement concerned urethral pressure, bladder pressure on voiding, PRUV and dysuria. The only drawback could be the onset or exacerbation of urinary incontinence.

Our trial also confirmed that the treatment is safe. A single instance of urethral bleeding was observed in our series. Schurch reported no side effects. ${ }^{11} \mathrm{We}$ did not note the effects reported by Dykstra (transitory paresis of the limbs, transitory exacerbation of 
autonomic dysreflexia) who used higher doses repeated three times at 1 week intervals. ${ }^{9}$

There can be several reasons for failure of treatment with BTx. In our series, the same dosage of BTx was used in all of the patients. This was done in an attempt to establish the efficacy of a low dose of BTx. This dose was insufficient for certain patients as was demonstrated in two by the success of a second injection with a higher dose. Another factor contributing to failure appeared, to us, to be poor bladder contractility (three cases), in accordance with the results of Schurch. ${ }^{11}$ Indeed, in spite of the diminution in DSD and urethral hypertonia after treatment with BTx, a low level of bladder contractility most probably does not permit improvement of bladder emptying. Another factor of failure reported by Schurch $^{11}$ was the presence of bladder neck dyssynergia, a parameter that was not quantified in our trial.

Our results show that low doses of BTx can be used initially to treat DSD. These doses should be increased if there is failure. Patient selection should include the degree of bladder contractility, since those with high contractility have better therapeutic success rates. Furthermore, Schurch's results ${ }^{11}$ suggest that one should look for bladder neck dyssynergia using a video-urodynamic system or a micturition-cystourethrogram during pretherapeutic work-up.

The duration of the effects of treatment after a single injection was 2 to 3 months on the average, in our trial as in the literature. ${ }^{9,11}$ This duration of action was the same as that of BTx in its other indications, ${ }^{6,7}$ the transitoriness of the effect being due to synaptic regrowth after injection. ${ }^{7}$ However, in two patients in our series, the clinical effect lasted beyond the usual duration of action of BTx, which might signify a more durable modification of the reflex status and urethral behaviour.

In this study, we used injections administered endoscopically according to the procedure described by Dykstra. ${ }^{9}$ Schurch et al ${ }^{11}$ carried out a comparative study of this procedure and transperineal injection using an electrode-needle. They found the efficacy of the two methods to be practically identical. It follows that the transperineal route, which does not require a urologist, a Foley catheter and prophylactic treatment of autonomic hyperreflexia, might in the future permit a simplification of this treatment and a reduction in its cost.

The price of BTx is high: 125 U.S. dollars for 150 I.U. Dysport BTx in France. The cost/efficacy ratio of this treatment is, in our opinion, more favourable when it permits the replacement of intermittent catheterization by condom catheter drainage associated with suprapubic tapping, especially when patients cannot perform the catheterizations themselves, ie principally in tetraplegic patients.

\section{Conclusion}

BTx thus appears to be a promising treatment of DSD for certain patients refractory to intermittent catheterization or surgery. The long-term capacity of BTx to protect the proximal urinary tract against the effects of DSD remains to be determined.

\section{References}

1 Kiesswetter $\mathrm{H}$, Schober $\mathrm{W}$. Lioresal in the treatment of neurogenic bladder dysfunction. Urol Int 1975; 30: 63-69.

2 Dmochowski RR, Ganabathi K, Leach GE. Non-operative management of the urinary tract in spinal cord injury. Neurourol Urodynamics 1995; 14: $47-55$.

3 Vapnek JM, Couillard DR, Stone AR. Is sphincterotomy the best management of the spinal cord injured bladder? J Urol 1994; 151: $961-964$.

4 Yang CC, Mayo ME. External urethral sphincterotomy: longterm follow up. Neurourol Urodynamics 1995; 14: 25 -31.

5 Brindley GS. The first 500 patients with sacral anterior root stimulator implants: general description. Paraplegia 1994; 32: $795-805$.

6 Grazko MA, Polo KB, Jabbari B. Botulinum toxin A for spasticity, muscle spasms, and rigidity. Neurology 1995; 45: $712-717$.

7 Jankovic J, Schwartz K, Donovan DT. Botulinum toxin in the treatment of cranial-cervical dystonia, spasmodic dysphonia, other focal dystonias and hemifacial spasm. J Neurol Neurosurg Psychiatry 1990; 53: 633-639.

8 Dykstra DD et al. Effects of botulinum A toxin on detrusorsphincter dyssynergia in spinal cord injury patients. J Urol 1988; 139: $919-922$.

9 Dykstra DD, Sidi AA. Treatment of detrusor-sphincter dyssynergia with botulinum A toxin: a double blind study. Arch Phys Med Rehabil 1990; 71: 24-26.

10 Schurch B et al. Effets de la toxine botulinique A sur le sphincter strié périurétral des vessies neurogènes. Etude préliminaire. J Urol 1990; 96: $375-380$.

11 Schurch B et al. Botulinum A toxin as a treatment of detrusorsphincter dyssynergia: a prospective study in 24 spinal cord injury patients. J Urol 1996; 155: 1023 - 1029.

12 Yalla SV, Rossier AB, Fam B. Dyssynergic vesicourethral responses during bladder rehabilitation in spinal cord patients: effects of suprapubic percussion, Crédé method and bethanechol chloride. J Urol 1976; 115: 575-579. 